\title{
Niveles de salud psicológica y laboral en practicantes de Pilates Levels of Psychological and Occupational Health in Pilates Adherents
}

\author{
Salvador Boix Vilella \\ Eva León Zarceño \\ Universidad Isabel I, Burgos, España \\ Universidad Miguel Hernández de Elche, España \\ Miguel Ángel Serrano Rosa \\ Universitat de València, España
}

\begin{abstract}
Resumen
Actualmente, la práctica de Pilates carece de fundamentación científica sólida que establezca beneficios sobre la salud psicológica. El objetivo principal del presente trabajo fue analizar si los participantes que combinan Pilates con otras Actividades Físico-Deportivas (AFD) presentaban puntuaciones más altas en las variables psicosociales y laborales estudiadas que practicantes de Pilates, practicantes de otras AFD diferentes al Pilates y participantes sedentarios. El segundo objetivo fue comprobar si los años de experiencia practicando Pilates, de forma aislada, influían positivamente sobre las variables estudiadas. El cuestionario de autoeficacia (AEG), autoestima (RSE), personalidad situacional (CPS), optimismo (LOT-R), satisfacción laboral (S10/12) y engagement (UWES) fueron completados por 212 participantes con una edad media de 41.83 años $(D T=12.41)$. Los practicantes de Pilates asistían a centros especializados en la técnica Pilates original. La metodología empleada fue de corte transversal. Las puntuaciones más altas en autoeficacia, estabilidad emocional, autoconcepto, optimismo y satisfacción con las prestaciones recibidas obtenidas por el grupo que combina Pilates con otras AFD no son estadísticamente significativas frente al resto de los grupos. Sin embargo, los minutos semanales de AFD correlacionan significativamente con la autoeficacia, el autoconcepto y la tendencia optimista. Los resultados del segundo objetivo muestran diferencias significativas en las variables autoeficacia y autoconcepto entre los practicantes más expertos de Pilates con respecto a los principiantes. Como conclusión, no se detectan diferencias significativas entre los grupos analizados, pero una práctica continuada y regular de Pilates en el tiempo, superior a 5 años, puede reportar interesantes beneficios psicológicos.
\end{abstract}

Palabras clave: ejercicio, método Pilates, autoeficacia, salud laboral, salud psicosocial.

Salvador Boix Vilella; Universidad Isabel I, Burgos, España; Eva León Zarceño; Universidad Miguel Hernández de Elche, España; Miguel Ángel Serrano Rosa; Universitat de València, España.

La correspondencia en relación con este artículo se dirige a Salvador Boix Villela, Universidad Isabel I, Burgos, España. Dirección electrónica: salvador.boix@uil.es 


\begin{abstract}
:
Currently, the practice of Pilates lacks a solid scientific foundation that establishes benefits on psychological health. The main objective of the present work was to analyze whether participants who combine Pilates with other Physical Sports Activities (PSA) had higher scores in psychosocial and work variables studied than Pilates adherents, devotees of other PSAs other than Pilates and sedentary participants. The second objective was to check whether years of experience practicing Pilates, in isolation, had a positive influence on the variables studied. Two hundred twelve participants completed the self-efficacy questionnaire (GSE), self-esteem (RSE), situational personality (CPS), optimism (LOT-R), job satisfaction (S10/12) and engagement (UWES) questionnaires with an average age of 41.83 years $(S D=12.41)$. Adherents of Pilates attended centers specialized in original Pilates techniques. The methodology used was cross-sectional. The highest scores on self-efficacy, emotional stability, self-concept, optimism and satisfaction with the benefits received by the group that combines Pilates with other PSAs are not statistically significant compared to the rest of the groups. However, the weekly minutes of PSA correlate significantly with self-efficacy, self-concept and optimistic tendency. The results of the second objective show significant differences in the self-efficacy and self-concept variables among the most expert Pilates adherents with respect to beginners. In conclusion, no significant differences were detected between the analyzed groups, but a continued and regular practice of Pilates over time (more than 5 years), may provide interesting psychological benefits.
\end{abstract}

Keywords: Exercise, Pilates Method, Self-Efficacy, Occupational Health, Psychosocial Health.

Numerosos trabajos han demostrado que la práctica regular de ejercicio físico tiene un impacto positivo sobre la salud, tanto a nivel físico como psicológico y social (Blacklock, Rhodes, \& Brown, 2007; Ekelund et al., 2012; Mikkelsen, Stojanovska, Polenakovic, Bosevski, \& Apostolopoulos, 2017). El ejercicio físico planificado y estructurado, además de la mejora de la condición física, potencia los recursos motores en beneficio de la salud psicosocial (Sánchez, Ureña, \& Garcés de Los Fayos, 2002; Stein, Molinero, Salguero, Corrêa, \& Márquez, 2013). Asimismo, combate el aislamiento, la depresión, la ansiedad y favorece la autoestima (García-Molina, Baeza, \& Fernández, 2010; Gómez-Juanes et al., 2015). Sin embargo, dentro de la comunidad científica, todavía no existe una evidencia sólida sobre los efectos del Pilates en la salud psicológica del practicante (Boix, León, \& Serrano, 2014; Fernández, Santana, \& Merino, 2011; Fleming \& Herring, 2018). Por ello, la mayoría de autores coinciden en que son necesarias nuevas investigaciones (Altan, Korkmaz, Bingol, \& Gunay, 2009; Bian et al., 2013; Curi, Vilaça, Haas, \& Fernandes, 2018; Stan, Collins, Olsen, Croghan, \& Pruthi, 2012), porque gran parte de los estudios publicados destacan por la escasa muestra (Boix, León, \& Serrano, 2017a).

Durante mucho tiempo, se ha sustentado la idea de que existía una relación directa entre cantidad de ejercicio físico y beneficios para la salud. Sin embargo, se cuestiona abiertamente la relación entre beneficios para la salud y las altas cantidades e intensidades de ejercicio físico (Pérez-Samaniego \& Devís, 2003). Desde la Organización Mundial de la Salud (OMS) se establecen, en el 2010, las recomendaciones mundiales sobre actividad física para la salud que son la base de los posteriores planes de ejercicio llevados a cabo por los países miembros. En ellos se acuerda que un adulto sano, de 18 a 64 años, debería participar como mínimo 150 minutos semanales de actividad física aeróbica, de intensidad moderada, o de 75 minutos de actividad física aeróbica vigorosa cada semana, o bien una combinación equivalente de 
actividades moderadas y vigorosas. Además, como mínimo, dos veces por semana, se deberían realizar actividades de fortalecimiento de los grandes grupos musculares.

Los beneficios del ejercicio físico también influyen positivamente en la salud laboral. Se reducen así los niveles de ausentismo y estrés laboral del trabajador (Ansoleaga-Moreno \& Miranda-Hiriart, 2014; Blanco-Álvarez \& Thoen, 2017; Kuoppala, Lamminpaa, \& Husman, 2008). En el mundo laboral, el estudio de los aspectos organizacionales, de los factores psicosociales y su relación con la salud laboral ha adquirido, en los últimos años, gran importancia y reconocimiento (Fraticelli, González, Uribe, Moreno, \& Orengo, 2018; Houdmont \& Leka, 2010; Näswall, Hellgren, \& Sverke, 2008). Ese mayor interés queda reflejado en la publicación de nuevos trabajos centrados en el estudio de dichos factores sobre la salud física, psíquica y/o social del trabajador (Raffo, Ráez, \& Cachay, 2013; Schaufeli \& Salanova, 2002). Los factores psicosociales comprenden las interacciones entre, por una parte, el trabajo, el medio ambiente y las condiciones de organización y, por otra, las capacidades del trabajador, sus necesidades, su cultura y su situación personal fuera del trabajo, todo lo cual, a través de sus percepciones y experiencias, pueden influir en su salud, su rendimiento y su satisfacción en el trabajo (Solanes, Martín del Río, Rodríguez, Benavides, \& Martínez, 2013). Entre las posibles estrategias para hacer frente a los riesgos psicosociales asociados al ámbito laboral sería importante incluir la actividad física, porque es la opción más simple y rentable para mejorar la salud, el bienestar y la productividad de los trabajadores (De Miguel, Schweiger, De las Mozas, \& Hernández, 2011). Una práctica continuada de ejercicio permitirá mejoras en la condición física, en el rango de grasa y en el Índice de Masa Corporal (IMC) del trabajador lo que incidirá positivamente en su salud y rendimiento (García-Martos, Calahorro, Torres-Luque, \& Lara, 2010; Gómez-Ambrosi et al., 2012). Además, podrá controlar mejor sus emociones negativas ante posibles eventos estresantes (Goyen \& Anshel, 1998) y estar más preparado para hacer frente a la adversidad (Bebetsos \& Antoniu, 2003). Por ello, se considera que la intervención en materia de salud laboral relacionada con el ejercicio físico debe entenderse como una herramienta que puede ser rentable a medio y largo plazo y no como un fin en si misma (Pérez-Marín, Yélamos, \& Rodríguez-Pérez, 2015).

Joseph Hubertus Pilates, creador del método Pilates, tuvo una infancia marcada por las enfermedades que le llevó a interesarse por el estudio y la práctica de diferentes actividades físicas hasta desarrollar su propio sistema de ejercicios al que denominó contrología (Pilates, 1934). Por contrología se entiende la ciencia y arte del desarrollo coordinado de la mente, el cuerpo y el espíritu, a través de movimientos naturales, bajo el estricto control de la voluntad (Pilates \& Miller, 1945). En el año 1926, Joseph Pilates abrió en Nueva York su primer estudio en el que emplea el desarrollo holístico para mejorar la salud de sus clientes en sus diferentes ámbitos físico, mental y social (Isacowitz, 2009). Tras su muerte, el método Pilates llegó a desaparecer del mercado por una serie de disputas legales. Finalmente, se permitió usar el nombre de Pilates en la razón comercial de cualquier estudio alcanzando en muy pocos años elevadas cotas de popularidad en todo el mundo (Boix, León, \& Serrano, 2017b). Actualmente, la concentración, la centralización, el control, la respiración, la precisión y la fluidez son los principios comunes que rigen la técnica Pilates en la mayoría de las escuelas (Isacowitz, 2009; Shipside, 2005). Otros centros deportivos se han alejado del método Pilates original con nuevas técnicas, como el yogalates o el aeroPilates, que alteran el orden de los ejercicios y la posición de la columna (Shea \& Moriello, 2014). A pesar de las diferentes modalidades existentes, hay trabajos bien fundamentados que han encontrado mejoras del 
método Pilates original sobre variables psicológicas como: la autoestima, depresión y calidad de vida en mujeres de 20 a 45 años (Küçük \& Livanelioglu, 2015); la autoeficacia (Boix et al., 2017b; Caldwell, Harrison, Adams, \& Triplett, 2009); el autoconcepto y la apariencia física de mujeres adultas (CruzFerreira et al., 2011). Según el modelo estructural de Memmedova (2015), se considera que el Pilates mejora los factores psicológicos. Se considera cada uno de ellos por separado, a partir del aumento de la fuerza, de la calidad del sueño, del flujo sanguíneo, de la relajación, etc.

En función de estos antecedentes, los objetivos que se plantean en este trabajo son los siguientes: (a) analizar si los participantes que combinan Pilates con otras Actividades Físico-Deportivas (AFD) presentan puntuaciones más altas en las variables psicosociales y laborales estudiadas (autoeficacia, autoestima, estabilidad emocional, autoconcepto, optimismo, satisfacción laboral y engagement) que practicantes de Pilates, practicantes de otras AFD diferentes al Pilates y participantes sedentarios; (b) comprobar si los años de experiencia practicando Pilates, de forma aislada, influyen positivamente sobre las variables estudiadas.

En función de los objetivos propuestos, y en consonancia con la actual tendencia en investigación, el presente trabajo pretende evaluar variables positivas dejando de lado otras más clásicas como la depresión o la ansiedad con el fin último de poder responder a las siguientes cuestiones: ¿Cuál de los cuatro grupos analizados presenta mayores niveles de salud psicológica y laboral? ¿Los participantes que combinan Pilates con otras AFD obtienen mejores resultados que aquellos que lo practican en exclusiva? ¿El grupo sedentario obtendrá las peores puntuaciones? ¿Los participantes con más años de experiencia practicando Pilates, de manera aislada o exclusiva, registran puntuaciones más altas sobre la salud psicosocial?.

\section{Método}

\section{Participantes}

La muestra total del estudio estaba formada por 212 participantes (171 mujeres y 41 hombres) con una edad media de 41.83 años $(D T=12.41)$. En el caso de las mujeres, la edad media registrada fue de $41.35(D T=12.50)$ y en los hombres fue de $43.88(D T=11.94)$. A partir de las respuestas obtenidas en un breve cuestionario sociodemográfico, de elaboración propia, se descartaron los participantes que estaban en tratamiento de enfermedades físicas y/o psicológicas graves y a aquellos que desarrollaban sus funciones en empresas que no estuvieran dentro del sector terciario o servicios. Este último criterio se debe a que los trabajadores pertenecientes a los sectores primario y secundario suelen estar expuestos a una actividad física laboral, no reglada, superior que podría tener influencia en los resultados finales por lo que se decide minimizar el impacto de dicha actividad incluyendo solo empleados del sector terciario. Respecto al tipo de actividad laboral, los participantes trabajan como profesor en educación secundaria $(19.33 \%)$, maestro de colegio (13.33\%), administrativo (6\%), comercial $(6 \%)$, empresario $(6 \%)$, enfermero $(5.33 \%)$, dependiente $(4.65 \%)$, médico $(4 \%)$, peluquero $(3.3 \%)$, economista $(2.66 \%)$, carnicero $(2.66 \%)$, educador $(2 \%)$, gestor $(2 \%)$, monitor $(2 \%)$, limpiador $(2 \%)$, fisioterapeuta $(2 \%)$, recepcionista $(2 \%)$, mercadólogo $(2 \%)$, empleado de banca $(2 \%)$, consultor $(1.33 \%)$, camarero $(1.33 \%)$, juez $(1.33$ $\%$ ), esteticista $(1.33 \%)$, informático (1.33\%), farmacéutico (1.33\%), frutero $(0.69 \%)$, logopeda $(0.69 \%)$, arquitecto $(0.69 \%)$ y modisto $(0.69 \%)$. Para abordar el primer objetivo, los participantes fueron divididos en cuatro grupos: practicantes de Pilates, practicantes de Pilates y otras AFD, practicantes de AFD 
diferente al Pilates y participantes sedentarios. El procedimiento de clasificación empleado, en el presente trabajo, para catalogar a los participantes en activos y sedentarios fue acorde con la literatura existente y lo utilizado en el eurobarómetro sobre deporte y actividad física (Special Eurobarometer, 2014). El primer grupo estaba formado por 57 participantes (48 mujeres $[M=40.24, D T=9.22]$ y 9 hombres $[M$ $=47.88, D T=12.08]$ ) que únicamente practicaban como actividad física el método Pilates. El grupo solo Pilates cuenta con una práctica media de 2.39 días por semana $(D T=.92)$ con una duración media semanal de 141 minutos. El segundo grupo estaba formado por 68 participantes ( 50 mujeres $[M=46.14$, $D T=11.63]$ y 18 hombres $[M=45.50, D T=10.54])$ que, además de Pilates, realizaban otras AFD. Los participantes de este grupo asistían a clases de Pilates una media de 2.46 días por semana $(D T=.97)$. Además, éste grupo también realiza una media de 3.71 días por semana de otras prácticas deportivas diferentes a Pilates $(D T=1.74)$. Se desconoce si, en el mismo día, algunos participantes, realizaban ambas actividades. Ello podría variar estos datos de frecuencia semanal, pero se tiene la garantía que la cantidad total de AFD semanal es de 482 minutos. Las AFD que practican los integrantes de este grupo Pilates son caminar (30.77\%), correr (13.98\%), pádel (8.39\%), ciclismo (8.39\%), natación (6.99\%), gimnasia de mantenimiento (6.29\%), spinning (4.19\%), yoga (2.79\%), tenis $(2.79 \%)$, golf (2.79\%), danza (2.09\%), equitación (1.39\%) y otras $(9.15 \%)$. El tercer grupo, AFD diferente al Pilates, lo formaban 43 participantes $(33$ mujeres $[M=36.94, D T=12.68]$ y 10 hombres $[M=51.99, D T=12.81])$ con una práctica media semanal de 3.30 días $(D T=1.28)$ y con una cantidad total de AFD de 280 minutos a la semana. Las AFD practicadas por el grupo activo no Pilates son caminar (25.92\%), correr $(22.22 \%)$, gimnasia de mantenimiento (14.81\%), spinning (11.12\%), pádel $(9.26 \%)$, ciclismo $(7.41 \%)$ y otras $(9.26 \%)$. Por último, el grupo sedentario lo formaban 44 participantes ( 40 mujeres $[M=41.06, D T=15.48]$ y 4 hombres $[M$ $=34.10, D T=9.09]$ ) que no realizaban AFD. Para abordar el segundo objetivo, se divide el grupo solo Pilates en función de sus años de práctica. Para su nueva agrupación, se sigue el criterio utilizado en el estudio sobre habilidades psicológicas en esgrimistas de Reche, Cepero y Rojas (2010) de establecer dos grupos: cinco o menos años de práctica y más de cinco años.

\section{Instrumentos}

\section{Cuestionario sociodemográfico}

Se utilizó un pequeño cuestionario sociodemográfico de elaboración propia que se les administró junto al resto de cuestionarios. Los participantes debían responder a algunas preguntas sobre su historial de salud, hábitos físico-deportivos, datos personales y laborales considerados relevantes para la formación de los grupos. Esta información se incluye en la tabla 1, donde se muestran los datos sociodemográficos y antropométricos obtenidos. El nivel socioeconómico percibido fue evaluado a partir de una escala de elaboración propia con un rango de respuesta de 0-10, que preguntaba sobre su formación y poder adquisitivo. En el caso del IMC y el rango de grasa, estos fueron calculados a partir de las variables sociodemográficas altura y peso utilizando la fórmula de Gómez-Ambrosi et al. (2012). 
Tabla 1

Aspectos sociodemográficos y antropométricos de los participantes

\begin{tabular}{|c|c|c|c|c|c|}
\hline & \multicolumn{2}{|c|}{ Grupo Pilates $(n=125)$} & \multicolumn{2}{|c|}{ Grupo no Pilates $(n=87)$} & \multirow[b]{2}{*}{ Total } \\
\hline & Pilates & $\begin{array}{c}\text { Pilates y } \\
\text { AFD }\end{array}$ & Sedentarios & Activos & \\
\hline & $(n=57)$ & $(n=68)$ & $(n=44)$ & $(n=43)$ & $(n=212)$ \\
\hline \multicolumn{6}{|l|}{ Puntuaciones medias } \\
\hline Edad & 41.44 & 45.97 & 38.30 & 39.44 & 41.83 \\
\hline N. ${ }^{o}$ hijos & 1.14 & 1.26 & 1.02 & 1.05 & 1.14 \\
\hline Nivel económico & 6.35 & 6.35 & 5.84 & 6.07 & 6.19 \\
\hline IMC & 22.20 & 23.44 & 24.29 & 24.08 & 23.41 \\
\hline Rango de grasa & 29.37 & 30.54 & 32.73 & 31.03 & 30.78 \\
\hline Tazas café/día & 1.25 & 1.38 & 1.11 & 1.19 & 1.25 \\
\hline Número cigarros/día & 2.44 & 1.32 & 1.11 & 2.23 & 1.76 \\
\hline \multicolumn{6}{|c|}{ Frecuencias y porcentajes } \\
\hline Mujer & $48(84.2)$ & $50(73.5)$ & $40(90.9)$ & $33(76.7)$ & $171(80.7)$ \\
\hline Hombre & $9(15.8)$ & $18(26.5)$ & $4(9.1)$ & $10(23.3)$ & $41(19.3)$ \\
\hline Problemas de salud & $18(31.6)$ & $18(26.5)$ & $5(11.4)$ & $6(14.0)$ & $47(22.2)$ \\
\hline Toma medicamento & $17(29.8)$ & $23(33.8)$ & $9(20.5)$ & $10(23.3)$ & $59(27.8)$ \\
\hline Trabaja & $42(73.7)$ & $50(73.5)$ & $30(68.2)$ & $28(65.1)$ & $150(70.8)$ \\
\hline Busca un trabajo & $5(8.8)$ & $4(5.9)$ & $6(13.6)$ & $7(16.3)$ & $22(10.4)$ \\
\hline No, no lo busco & $10(17.5)$ & $14(20.6)$ & $8(18.2)$ & $8(18.6)$ & $40(18.9)$ \\
\hline
\end{tabular}

\section{Autoeficacia General (AEG)}

La autoeficacia se considera un factor muy importante para el bienestar de las personas y las bases de datos científicas muestran que dicha variable ha sido objeto de múltiples investigaciones. Para medir la autoeficacia se ha utilizado la escala adaptada para población española desarrollada por Baessler y Schwarzer (1996). Esta prueba está formada por diez ítems donde los participantes deben responder a cada enunciado mediante una escala tipo Likert con cuatro alternativas de respuesta. La escala mide de forma directa la percepción de autoeficacia general. La escala ya ha sido utilizada ampliamente en población española. Sus propiedades psicométricas resultan adecuadas para su uso (Martín et al., 2002). El índice de fiabilidad alfa de Cronbach recogido en el trabajo de Rueda y Pérez-García (2004) fue de 90 y en el presente trabajo fue de .85 . 


\section{Autoestima (RSE)}

La autoestima es una variable de gran interés clínico por su relación con el bienestar general y muy particularmente con cuadros como la depresión (Rosenberg, 1965). La escala aquí utilizada, fue desarrollada por Rosenberg (1965) e incluye diez afirmaciones que aluden a sentimientos globales de autovaloración. Los ítems se responden en una escala de cuatro puntos y la mitad de los ítems están redactados en sentido positivo y la otra mitad en sentido negativo. La puntuación total oscila entre 10 y 40 . McCarthy y Hoge (1982) han informado de coeficientes de consistencia (alfa de Cronbach) que se sitúan entre .74 y .77. En el presente estudio, el alfa de Cronbach obtenido fue .85. La validez de la escala como medida unidimensional de la autoestima ha sido también comprobada por Vázquez, Jiménez y Vázquez-Morejón (2004).

\section{Escalas psicológicas del Cuestionario de Personalidad Situacional (CPS)}

El Cuestionario de Personalidad Situacional fue elaborado en España, por Fernández-Seara, Seisdedos y Mielgo (2001). Permite evaluar 17 escalas de personalidad de gran interés práctico en psicología aplicada. En este estudio se utilizan (a) la escala estabilidad emocional que aprecia el ajuste general de las emociones y los afectos: el control y la estabilidad emocional; (b) la escala autoconcepto que alude a la valoración personal que uno hace de sí mismo a partir de la percepción de su propia valía personal. El cuestionario total consta de 233 elementos de tipo verdadero-falso y es de breve y fácil aplicación. Para obtener la fiabilidad test-retest se realizaron dos aplicaciones donde ninguno de los coeficientes baja del valor .88. Una de las dos escalas más fiables es la estabilidad emocional con un coeficiente .94 (Gutiérrez, Flores, \& Gibbons, 2001). En el presente estudio, la escala estabilidad emocional obtuvo un índice alfa de Cronbach de .79 y la escala autoconcepto de .74. El CPS se ha estudiado en diferentes muestras, condiciones y correlacionado con otras medidas psicológicas relacionadas poniendo de manifiesto una notable validez de constructo (Fernández-Seara, Seisdedos, \& Mielgo, 2008).

\section{Revised Life Orientation Test (LOT-R)}

La variable optimismo adquiere mayor relevancia en investigación con el avance de lo que se conoce como Psicología positiva que abarca enfoques más positivos y optimistas de la salud mental. Para medir el optimismo disposicional o predisposición a las expectativas de resultados positivos o negativos, se utilizó el Test Revisado de Orientación Vital (Scheier, Carver, \& Bridges, 1994) en la versión española desarrollada por Otero, Luengo, Romero, Gómez y Castro (1998). El LOT-R consta de seis ítems (más cuatro ítems que no evalúan optimismo), tres están redactados en sentido positivo (dirección optimismo) y tres en sentido negativo (dirección pesimismo). Los ítems redactados en sentido negativo se revierten y se obtiene una puntuación total orientada hacia el polo de optimismo (optimismo total). En cuanto a las propiedades psicométricas de la versión española el estudio de García-Naveira y Díaz-Morales (2010) obtiene una fiabilidad alfa de Cronbach de .74 para optimismo y .70 para pesimismo. En el caso del presente trabajo, las puntuaciones fueron de .73 para optimismo y .70 para pesimismo.

\section{Satisfacción laboral (S10/12)}

La satisfacción laboral es uno de los aspectos a los que se le ha prestado más atención dentro de las organizaciones. El cuestionario de Meliá y Peiró (1989) se compone de 12 ítems y mide tres factores: satisfacción con el ambiente físico, satisfacción con la supervisión y satisfacción con las prestaciones 
recibidas. El primer factor abarca el espacio de trabajo, la limpieza, la higiene y la temperatura; el segundo factor se relaciona con la forma y la justicia en que los superiores juzgan la tarea del empleado y el tercer factor hace referencia al grado en que la empresa cumple con las leyes laborales (Meliá \& Peiró, 1989). Las alternativas de respuesta se registran en una escala de siete grados donde uno corresponde a muy insatisfecho y siete a muy satisfecho. El S10/12 fue extraído del estudio de dos versiones anteriores el S4/82 y el S20/23. La versión S10/12 del cuestionario de satisfacción mantiene una consistencia interna con un Alfa de Cronbach de .88 y unos niveles de validez que mejoran la escala original (Meliá y Peiró, 1989). Los valores alfa de Cronbach obtenidos en el presente trabajo fueron de .82 para el factor ambiente físico, de .87 para el factor supervisión y de .77 para las prestaciones recibidas.

\section{Utrecht Work Engagement Scale (UWES)}

Dentro de la Psicología Organizacional, el engagement se considera un factor importante para lograr organizaciones saludables. Para valorarlo, se utilizó la versión española (Salanova, Schaufeli, Llorens, Peiró, \& Grau, 2000) del Utrecht Work Engagement Scale (Schaufeli, Salanova, GónzalezRomá, \& Bakker, 2002). La escala está compuesta por 17 ítems distribuidos en tres factores: vigor, dedicación y absorción. Las respuestas se registran en una escala tipo Likert de siete posibles respuestas, donde cero corresponde a nunca y seis a siempre/todos los días. Los coeficientes alfa de Cronbach que se han obtenido en el estudio de Fajardo, Iroz, López y Mirón (2013) para las tres dimensiones son: .80 (vigor) .80 (absorción) y .85 (dedicación). Las puntuaciones alfa de Cronbach obtenidas en el presente estudio fueron de .81 para vigor, de .84 para absorción y de .86 para dedicación.

\section{Procedimiento}

El diseño de investigación utilizado es empírico mediante la estrategia descriptiva-comparativa. La metodología empleada en esta investigación fue de corte transversal. Se concretó una reunión con los dirigentes de una franquicia de Pilates en España para poder explicar los objetivos del estudio y poder reclutar a sus alumnos para participar en la investigación. Una vez que aceptaron colaborar en el trabajo, se concertaron reuniones en cada centro al explicar el funcionamiento a profesores y alumnos. Todo el profesorado, de los centros participantes en el estudio, fue preparado en la misma escuela de formación de Pilates por un mismo grupo de docentes. Además, la metodología empleada por los profesores en las diferentes aulas de Pilates era la misma, porque todos los centros franquiciados se basaban en una programación mensual común. En definitiva, se trató de que todas las personas que acudían a clases de Pilates, independientemente del centro, participaran del método Pilates original con una organización y metodología común. También, se estableció que todos los sujetos fueran mayores de 18 años y que pudieran participar tanto hombres como mujeres siempre que firmaran una hoja de consentimiento informado una vez leídos los fines y los objetivos del estudio. En el caso del grupo activo no Pilates y del grupo sedentario, se mantuvieron los mismos criterios de inclusión salvo la asistencia a clases de Pilates. Estos participantes asistían a centros, situados en las mismas provincias que el grupo Pilates, de una franquicia de formación de idiomas, nuevas tecnologías, administración, actividades físicas y deportivas, hostelería, etc. Los cuestionarios fueron cumplimentados en el mes de febrero del año 2013 en un tiempo máximo de 30 minutos. Todos los participantes, tanto practicantes o no de Pilates completaron los cuestionarios en horario de tarde dentro de un ambiente tranquilo y bajo la supervisión de uno de 
los investigadores en las diferentes salas de cada centro. La participación fue totalmente voluntaria y se garantizaba la privacidad y confidencialidad de sus respuestas. Así, se cumplieron los principios éticos de la última reforma de la Declaración de Helsinki (1964) aprobada en Fortaleza (2013).

\section{Análisis estadístico}

En primer lugar, se calcularon los valores atípicos para las todas las variables. Se consideraron atípicas las puntuaciones $Z$ mayores de 3 o menores a -3. Se excluyeron, por tanto, a siete participantes. En segundo lugar, se realizó la prueba de Kolmogorov-Smirnoff para comprobar la normalidad de las variables utilizadas. Una vez depurada la base de datos, se realizaron análisis descriptivos y de frecuencias. Se utilizó el modelo lineal general univariante, ANOVAS, para la comparación de las medias de las variables sociodemográficas en los cuatro grupos establecidos. Las variables edad, IMC y minutos de actividad física semanal resultaron significativas entre grupos por lo que se trataron como covariadas en los análisis de las ANCOVAS. También, se averiguaron las comparaciones múltiples de Bonferroni para las pruebas post-hoc y las estimaciones del tamaño del efecto eta cuadrado $\eta^{2}$. En el caso de que alguna de las variables covariadas resultara significativa, se estudió la dirección de su influencia mediante correlaciones de Pearson. El nivel de significación estadística fue establecido en $p<.05$. Para el procesamiento estadístico de los datos, se ha usado el paquete estadístico SPSS para Windows en su versión 20.0.

\section{Resultados}

En relación con el objetivo número uno, se toma como variable de agrupación la resultante de la división de los 212 participantes en los cuatro subgrupos establecidos. En el caso de las variables psicológicas y laborales analizadas (autoeficacia, autoestima, estabilidad emocional, autoconcepto, optimismo, prestaciones recibidas, supervisión, ambiente físico, vigor, dedicación y absorción), se consideran como dependientes en los análisis de diferencias de medias. En estos primeros análisis, se debe tener en cuenta que las variables sociodemográficas minutos semanales de actividad física, la edad y el IMC fueron tratados como covariadas. El valor de las ANCOVAS no muestra diferencias estadísticamente significativas en ninguna de las variables psicosociales y laborales analizadas. A pesar de ello, los estadísticos descriptivos obtenidos por cada grupo muestran que la combinación de Pilates con otras AFD obtiene puntuaciones más altas en el apartado de salud psicológica, a excepción de la autoestima, respecto al resto de grupos. En el apartado laboral, teniendo nuevamente presente la ausencia de diferencias significativas, es el grupo solo Pilates el que acumula mayores puntuaciones, salvo en prestaciones recibidas, frente al resto de grupos. Véase tabla 2. 
Tabla 2

Niveles de significación, puntuaciones medias y desviaciones típicas en función de la actividad o actividades fisicas realizadas

\begin{tabular}{|c|c|c|c|c|c|c|}
\hline \multirow[b]{2}{*}{ Variable } & \multicolumn{2}{|c|}{ Grupo Pilates } & \multicolumn{2}{|c|}{ Grupo no Pilates } & \multirow[t]{2}{*}{ Total } & \multirow{2}{*}{$\frac{\text { Ancova }}{p}$} \\
\hline & Pilates & Pilates y AFD & Sedentario & Activo & & \\
\hline Autoeficacia & $.48(.05)$ & $.50(.07)$ & $.46(.06)$ & $.50(.05)$ & $.49(.06)$ & .77 \\
\hline Autoestima & $1.78(.46)$ & $1.74(.54)$ & $1.86(.46)$ & $1.74(.53)$ & $1.78(.50)$ & .93 \\
\hline $\begin{array}{l}\text { Estabilidad } \\
\text { emocional }\end{array}$ & $40.58(4.92)$ & $40.96(4.54)$ & $39.02(5.15)$ & $40.67(4.31)$ & $40.40(4.75)$ & .76 \\
\hline Autoconcepto & $1.61(.04)$ & $1.62(.04)$ & $1.60(.05)$ & $1.62(.04)$ & $1.61(.04)$ & .28 \\
\hline Tendencia optimista & $4.07(3.47)$ & $5.96(3.88)$ & $2.14(4.39)$ & $3.93(3.84)$ & $4.25(4.09)$ & .17 \\
\hline Vigor & $5.08(.58)$ & $4.87(.76)$ & $4.82(.69)$ & $5.00(.96)$ & $4.94(.74)$ & .39 \\
\hline Dedicación & $.66(.12)$ & $.62(.16)$ & $.66(.09)$ & $.63(.18)$ & $.64(.14)$ & .22 \\
\hline Absorción & $3.95(1.25)$ & $3.63(1.32)$ & $3.98(1.15)$ & $3.65(1.38)$ & $3.79(1.28)$ & .29 \\
\hline Supervisión & $.76(.13)$ & $.74(.14)$ & $.71(.14)$ & $.71(.22)$ & $.73(.15)$ & .48 \\
\hline Ambiente físico & $.76(.10)$ & $.75(.09)$ & $.75(.06)$ & $.70(.16)$ & $.74(.11)$ & .18 \\
\hline $\begin{array}{l}\text { Prestaciones } \\
\text { recibidas }\end{array}$ & $.69(.18)$ & $.72(.17)$ & $.66(.19)$ & $.61(.25)$ & $.68(.20)$ & .19 \\
\hline
\end{tabular}

De las tres variables covariadas, únicamente los minutos semanales de ejercicio resultaron significativos en las variables psicosociales y laborales analizadas. Una vez detectada esa diferencia significativa, se exploró la dirección de esta relación mediante correlaciones de Pearson. La significación de la variable minutos de ejercicio se descubrió en las variables autoeficacia $(p=.036)$, autoconcepto $(p=.031)$ y tendencia optimista $(p=.043)$. Las correlaciones de Pearson llevadas a cabo mostraron una relación directa y significativa entre minutos de AFD semanal y las variables autoeficacia $(p=.001, r=249)$, autoconcepto $(p=.006, r=189)$ y optimismo $(p=.001, r=320)$. En definitiva, un mayor número de minutos de actividad física semanal se relaciona con mejores puntuaciones en las variables psicológicas descritas.

En relación con el objetivo número dos, se exponen los resultados relativos a los años de experiencia practicando exclusivamente Pilates. En este caso, las pruebas $t$ student muestran diferencias significativas entre los dos grupos establecidos, cinco o menos años de práctica de Pilates y más de cinco años, en la variable sociodemográfica minutos totales de Pilates $(p=.001)$. Dicha variable ha sido tratada como covariada en los análisis de ANCOVAS que muestran diferencias significativas en las variables autoeficacia $\left(F_{1,55}=4.43, p<.040 \eta_{\mathrm{p}}^{2}=.074\right.$, power $\left.=.543\right)$ y autoconcepto $\left(F_{1,55}=5.07, p<.028 \eta_{\mathrm{p}}^{2}\right.$ $=.084$, power $=.600)$. En los dos casos, el grupo que lleva practicando más de cinco años obtiene más autoconcepto y autoeficacia según los análisis post-hoc Bonferroni y los estadísticos descriptivos. Los valores obtenidos en eta cuadrado y power indican cómo de fuerte es el efecto, ya que los valores de $p$ ( $p=.040$ para autoeficacia y $p=.028$ para autoconcepto) pueden verse afectados por el tamaño de la 
muestra y otros factores. En el presente estudio, los valores de eta y power son bajos, sobre todo los de eta, por lo que no se puede afirmar que tienen un importante efecto a pesar de ser significativo.

\section{Discusión}

El objetivo principal de este trabajo buscaba comparar los niveles de salud psicológica y laboral que presentaban los cuatro grupos analizados, dos grupos participantes de la técnica Pilates y dos grupos no practicantes de Pilates. Pese a la ausencia de diferencias estadísticamente significativas, entre los grupos, se observa cómo el grupo sedentario es el que obtiene las puntuaciones más bajas en autoeficacia, estabilidad emocional, autoconcepto, tendencia optimista, vigor y dedicación. En el caso del grupo activo no Pilates, se obtienen los registros más bajos de autoestima y satisfacción laboral. Por último, las puntuaciones más bajas en dedicación y absorción en el trabajo recaen en el grupo Pilates y otras AFD. Desde el punto de vista opuesto, el grupo solo Pilates obtiene los mayores niveles de salud laboral, con la excepción de la variable prestaciones recibidas, y el grupo Pilates y otras AFD registra los mayores niveles de salud psicológica, con la excepción de la variable autoestima. Las aportaciones del presente estudio no coinciden con los resultados de investigaciones previas que han señalado que los practicantes de Pilates registran mayores niveles de autoeficacia general (Boix et al., 2017b; Caldwell et al., 2009) y obtienen mejoras comportamentales en el estado de salud emocional percibido (García-Pastor, 2009). Tampoco los resultados relativos a la variable autoestima en los grupos Pilates coinciden con los obtenidos previamente en el trabajo de Küçük y Livanelioglu (2015). Lo mismo sucede con la variable autoconcepto que no obtiene puntuaciones significativamente superiores en los grupos Pilates como así sucede en el estudio de Cruz-Ferreira et al. (2011). En el presente trabajo, las diferencias significativas de autoeficacia, autoconcepto y tendencia optimista encontradas aparecen cuando se relacionan dichas variables con los minutos semanales de ejercicio físico. Estos resultados apuntan que a mayores cantidades de AFD mayores beneficios sobre la salud podrían obtenerse independientemente del tipo de actividad realizada.

En la línea de los resultados encontrados, el trabajo de Pérez-Samaniego y Devís (2003) cuestiona abiertamente la relación entre beneficios para la salud y altas cantidades e intensidades de actividad física. En relación con la dosis de actividad física adecuada, la Organización Mundial de la Salud (OMS, 2010) estableció unas recomendaciones mundiales muy generales sobre actividad física para la salud. Sin embargo, Pancorbo y Pancorbo (2011) señalan que la dosis exacta dependerá de la frecuencia, la intensidad, el tiempo y el tipo de ejercicio al atender a los principios de individualidad, progresión y mantenimiento del ejercicio. En este sentido, los autores del presente estudio creen plausible que la relación entre actividad física y salud tenga su explicación a partir de la escala de esfuerzo de Borg (1982) que permite medir el esfuerzo percibido del participante y así regular adecuadamente la intensidad y la carga física a sus necesidades individuales.

En las escalas laborales analizadas, los resultados encontrados no coinciden con el trabajo de Kloubec (2005) que sí encuentra mejoras en la satisfacción laboral al analizar el efecto del método Pilates. El trabajo de Boix, León y Serrano (2015) revela diferencias significativas al estudiar la satisfacción laboral de un grupo de trabajadores que se ejercita con Pilates suelo y máquinas frente a otros participantes que practican exclusivamente la modalidad Pilates suelo. Sin embargo, hay que tener en cuenta que la salud laboral es muy dependiente del contexto. Por ello, más allá de los resultados obtenidos en 
el presente estudio se sigue insistiendo en la idea de apoyar la práctica de actividad física para mejorar los niveles de salud psicosocial y laboral de empleados y directivos. De hecho, en entornos laborales las intervenciones que se realizan no suelen incluir la actividad física. Por ello, se considera interesante llevar a cabo programas de actividad física dentro de la misma empresa (De Miguel et al., 2011; Serrano y Boix, 2012) o en espacios públicos como facilitador de bienestar y salud mental (García-Martos et al., 2010; Leandro-Rojas, 2014).

Se debe tener muy en cuenta la elevada prevalencia del sedentarismo en la sociedad y que los minutos de inactividad física parecen actuar como factores de riesgo independientes para la salud (GonzálezGross \& Meléndez, 2013). Sin embargo, en el presente trabajo, que cuenta con un grupo sedentario, no se dan diferencias significativas. Esta ausencia de diferencias podría tener su explicación en la distribución grupal de los participantes. El estudio no ha evaluado la cantidad de actividad física por medio de un acelerómetro y existe toda una gama de actividades físicas, no regladas, que no se han tenido en cuenta para la formación de los grupos. Pese a tratar de minimizar las diferencias de actividad física con trabajadores del mismo sector, algunos sujetos considerados sedentarios no lo serían realmente si, por ejemplo, van caminando al trabajo todos los días. A pesar de la ausencia de resultados significativos, se sigue insistiendo en la línea de los investigadores Ooijendijk, Hildebrandt y Hopman-Rock (2006) que consideran necesario practicar actividad física casi todos los días de la semana para poder así obtener beneficios sobre la salud. Respecto a la duración del ejercicio, el United States Department of Health and Human Services (2008) recomienda un mínimo de 30 minutos al día. Dicho criterio lo cumplen ambos grupos de Pilates. Si bien, otro estudio remarca la necesidad de una práctica diaria superior a 60 minutos, puesto que aporta beneficios no alcanzables con un tiempo menor de ejecución (Bohórquez, Lorenzo, \& García, 2013). En resumen, el presente trabajo recalca la importancia de realizar AFD, reglada y/o no reglada, adaptada a las características individuales de cada uno para mantener la salud.

Por lo que respecta a los años de experiencia practicando Pilates, las publicaciones existentes apuntan a que la actividad física practicada regularmente debe mantenerse de manera frecuente en el tiempo para que los efectos sean visibles y de cierta duración (García-Martos et al., 2010; McAuley, Courneya, Rudolph, \& Lox, 1994). Los resultados del presente trabajo así lo atestiguan, pues los participantes del grupo solo Pilates con más de cinco años de práctica son quienes presentan más autoconcepto y autoeficacia frente a los más inexpertos. Estos resultados coinciden con otras publicaciones que relacionaron los años de experiencia en otros ámbitos físico-deportivos diferentes al Pilates (Bebetsos \& Antoniu, 2003; Goyen \& Anshel, 1998; Hanton, Neil, Mellalieu, \& Fletcher, 2008; Martín, 2003). Pese a que el entrenamiento de los integrantes del grupo solo Pilates no se ajusta perfectamente a todas las recomendaciones marcadas por la OMS, una práctica continuada y regular superior a cinco años parece influir positivamente sobre el autoconcepto y la autoeficacia.

Respecto a las limitaciones del estudio, cabe destacar que al no realizar un seguimiento de los participantes en el tiempo se tiene un conocimiento más limitado sobre los hábitos de vida y laborales de la muestra. También, hay una limitación en la distribución de los grupos, porque debería haberse evitado incluir diferentes prácticas de AF no Pilates en un mismo grupo. En el caso del grupo Pilates, los participantes sí asistían a centros especializados que organizan sus clases y forman a sus profesores atendiendo al método original que desarrolló Joseph Pilates dotando a la muestra de gran homogeneidad y superando 
la principal limitación detectada en las investigaciones previas (Boix et al., 2014). En relación con los instrumentos empleados, resultaría interesante que futuros trabajos consideren el uso de factores disposicionales en lugar de otros más estables como los utilizados en el presente trabajo. También, hubiera sido valioso registrar la hora del día en la que los participantes realizan AFD por lo que futuros estudios deberían analizar estos datos. Por último, podría existir una inferencia elevada ya que pese a la utilización del cuestionario sociodemográfico para detectar posibles patologías crónicas o enfermedades graves no se ha contado con información objetiva de historiales médicos. De cara a futuras investigaciones, se plantea la necesidad de superar estas limitaciones y seguir relacionando el método Pilates con variables psicológicas y laborales para tratar de aumentar la salud mental y la salud laboral de los trabajadores.

Como conclusión, se exponen las diferentes respuestas a las cuestiones planteadas a lo largo del presente estudio. Según los niveles de salud, los análisis descriptivos muestran como los participantes que combinan Pilates con otras AFD obtienen puntuaciones más altas en el apartado de salud psicológica y el grupo solo Pilates es el que acumula mayores puntuaciones en salud laboral. La ausencia de resultados significativos evidencia que la combinación del método Pilates con otras AFD no genera mayores beneficios para la salud de los participantes. En el caso del grupo sedentario, sus participantes acumulan un mayor número de variables con las puntuaciones más bajas. Por último, se ha comprobado que la práctica en exclusiva del método Pilates puede reportar interesantes beneficios psicológicos, tras cinco años de práctica, si se lleva a cabo de forma continuada y regular en el tiempo.

\section{Referencias}

Altan, L., Korkmaz, N., Bingol, Ü., \& Gunay, B. (2009). Effect of Pilates training on people with fibromyalgia syndrome: A pilot study. Archives of Physical Medicine and Rehabilitation, 90(12), 1983-1988. doi: 10.1016/j.apmr.2009.06.021

Ansoleaga-Moreno, E., \& Miranda-Hiriart, G. (2014). Depresión y condiciones de trabajo: Revisión actualizada de la investigación. Revista Costarricense de Psicología, 33(1), 1-14.

Asociación Médica Mundial. (1964). Declaración de Helsinki de la Asociación Médica Mundial. Principios éticos para las investigaciones médicas en seres humanos (64 Asamblea General, Fortaleza, 2013). Recuperado de https://www.wma.net/es/policies-post/declaracion-de-helsinki-de-la-amm-principios-eticos-para-lasinvestigaciones-medicas-en-seres-humanos/\#

Baessler, J., \& Schawarzer, R. (1996). Evaluación de la autoeficacia: Adaptación española de la Escala de Autoeficacia General. Ansiedad y Estrés, 2(1), 1-7.

Bebetsos, E., \& Antoniou, P. (2003). Psychological skills of Greek badminton athletes. Perceptual and Motor Skills, 97(3), 1289-1296.

Bian, Z., Sun, H., Lu, C., Yao, L., Chen, S., \& Li, X. (2013). Effect of Pilates training on alpha rhythm. Computational and Mathematical Methods in Medicine, 2013, Article ID 295986. doi: http://dx.doi. org $110.1155 / 2013 / 295986$

Blacklock, R. E., Rhodes, R. E., \& Brown, S. G. (2007). Relationship between regular walking, physical activity, and health-related quality of life. Journal of Physical Activity and Health, 4(2), 138-152. 
Blanco-Álvarez, T. M., \& Thoen, M. A. (2017). Factores asociados al estrés laboral en policías penitenciarios costarricenses. Revista Costarricense de Psicología, 36(1), 45-59.

Bohórquez, M. R., Lorenzo, M., \& García, A. J. (2014). Actividad física como promotor del autoconcepto y la independencia personal en personas mayores. Revista Iberoamericana de Psicología del Ejercicio y el Deporte, 9(2), 533-546.

Boix, S., León, E., \& Serrano, M. A. (2014). ¿Hay beneficios psicosociales por la práctica Pilates? Un análisis de la literatura científica. Cuadernos de Psicología del Deporte, 14(3), 117-128.

Boix, S., León, E., \& Serrano, M. A. (Octubre, 2015). Niveles de satisfacción laboral en función de la modalidad de Pilates practicada. Sesión de cartel presentado en el IX Congreso Hispano-Luso Psicología del Deporte, Madrid.

Boix, S., León, E., \& Serrano, M. A. (2017a). Evidencias de la práctica Pilates sobre la salud mental de personas sanas. Universidad y Salud, 19(2), 301-308. doi: http://dx.doi.org/ 10.22267/rus.171902.92

Boix, S., León, E., \& Serrano, M. A. (2017b). Identificación con el ejercicio físico y autoeficacia: Diferencias entre practicantes de Pilates vs no practicantes. Revista Iberoamericana de Psicología del Ejercicio y el Deporte, 12(1), 99-106.

Borg, G. (1982). Psychophysical bases of perceived exertion. Medicine \& Science in Sports \& Exercise, 14(5), 377281. doi: 10.1249/00005768-198205000-00012

Caldwell, K., Harrison, M., Adams, M., \& Triplett, T. (2009). Effects of Pilates and taiji quan training on selfefficacy, sleep quality, mood, and physical performance of college students. Journal of Bodywork and Movement Therapies, 13(2), 155-163. doi: 10.1016/j.jbmt.2007.12.001

Cruz-Ferreira, A., Fernandes, J., Gomes, D., Bernardo, L. M., Kirkcaldy, B. D., Barbosa, T., \& Silva, A. (2011). Effects of pilates-based exercise on life satisfaction, physical self-concept and health status in adult women. Women and Health, 51(3), 240-255. doi: 10.1080/03630242. 2011.563417

Curi, V. S., Vilaça, J., Haas, A. N., \& Fernandes, H. M. (2018). Effects of 16-weeks of Pilates on health perception and sleep quality among elderly women. Archives of Gerontology and Geriatrics, 74, 118-122. doi: http:// dx.doi.org/10.1016/j.archger.2017.10.012

De Miguel, J. M., Schweiger, I., De las Mozas, O., \& Hernández, J. M. (2011). Efecto del ejercicio físico en la productividad laboral y el bienestar. Revista de Psicología del Deporte, 20(2), 589-604.

Ekelund, U., Luan, J., Sherar, L. B., Esliger, D. W., Griew, P., \& Cooper, A. (2012). Moderate to vigorous physical activity and sedentary time and cardiometabolic risk factors in children and adolescents. Journal of the American Medical Association, 307(7), 704-712. doi: 10.1001/jama.2012.156

Fajardo, M., Iroz, M., López, D., \& Mirón, S. (2013). Relación entre engagement y las creencias de autoeficacia en los trabajadores. Revista Electrónica de Investigación en Docencia Creativa, 2, 79-92.

Fernández, E., Santana, F., \& Merino, R. (2011). Joseph Hubertus Pilates; anatomía de un gigante olvidado. Trances, 3(3), 353-378. 
Fernández-Seara, J. L., Seisdedos, C. N., \& Mielgo, M. (2001). CPS. Cuestionario de personalidad situacional. Madrid: Tea Ediciones.

Fernández-Seara, J. L., Seisdedos, C. N., \& Mielgo, M. (2008). CPS. Cuestionario de personalidad situacional. Manual. Madrid: TEA ediciones.

Fleming, K. M., \& Herring, M. P. (2018). The effects on mental health outcomes: A meta-analysis of controlled trials. Complementary Therapies in Medicine, 37, 80-95. doi: https://doi.org/10.1016/j.ctim.2018.02.003

Fraticelli, F. R., González, R. O., Uribe, A. F., Moreno, I., \& Orengo, J. C. (2018). Investigación: Diseño, construcción y validación de una escala para medir los factores psicosociales y la sintomatología orgánica en el área laboral. Informes Psicológicos, 18(1), 95-112. doi: http://dx.doi.org/10.18566/nfpsic.v18n1a05

García-Martos, M., Calahorro, F., Torres-Luque, G., \& Lara, A. J. (2010). Efectos de un programa de entrenamiento mixto sobre la condición física en mujeres jóvenes con sobrepeso. Cuadernos de Psicología del Deporte, 10(2), 11-16.

García-Molina, A., Baeza, A., \& Fernández, M. (2010). Beneficios de la actividad física en personas mayores. Revista Internacional de Medicina y Ciencias de la Actividad Física y del Deporte, 10(40), 556-576.

García-Naveira, A., \& Díaz-Morales, J. F. (2010). Relación entre optimismo/pesimismo disposicional, rendimiento y edad en jugadores de fútbol de competición. Revista de Iberoamericana de Psicología del Ejercicio y el Deporte, 5(1), 45-60.

García-Pastor, T. (2009). Efecto de la práctica del método Pilates: Beneficios en estado de salud, aspectos físicos y comportamentales (Tesis doctoral). Universidad de Castilla la Mancha, Toledo.

Gómez-Ambrosi, J., Silva, C., Galofré, J. C., Escalada, J., Santos, S., Millán, D., \& Frühbeck, G. (2012). Body mass index classification misses subjects with increased cardiometabolic risk factors related to elevated adiposity. International Journal of Obesity, 36(2), 286-294. doi: 10.1038/ijo.2011.100

Gómez-Juanes, R., Gili, M., Roca, M., Castro, A., López-Navarro, E., Crespi, C., \& García-Toro, M. (2015). Prescripción de ejercicio físico en la depresión por parte de Médicos de Familia. Factores involucrados. Revista de Psicología del Deporte, 24(1), 61-69.

González-Gross, M., \& Meléndez, A. (2013). Sedentarism, active lifestyle and sport: Impact on health and obesity prevention. Nutrición Hospitalaria, 28(5), 89-98.

Goyen, M. J., \& Anshel, M. H. (1998). Sources of acute competitive stress and use of coping strategies as a function of age and gender. Journal of Applied Developmental Psychology, 19(3), 469-486. doi: 10.1016/ S0193-3973(99)80051-3

Gutiérrez, J. R., Flores, H. M., \& Gibbons, P. (2001). El perfil psicosocial del agresor sexual en El Salvador. San Salvador: Universidad Tecnológica de El Salvador

Hanton, S., Neil, R., Mellaliu, S. D., \& Fletcher, D. (2008). Competitive experience and performance status: An investigation into multidimensional anxiety and coping. European Journal of Sport Science, 8(3), 143-152. 
Houdmont, J., \& Leka, S. (2010). Future directions in Occupational Health Psychology. En S. Leka \& J. Houdmont (Eds.), Occupational Health Psychology, (pp. 298-321). Oxford: Wiley-Blackwell.

Isacowitz, R. (2009). Pilates. Manual completo del método Pilates. Badalona: Paidotribo.

Kloubec, A. J. (2005). Pilates exercises for improvement of muscle endurance, flexibility, balance and posture (Tesis doctoral). University of Minnesota, Minnesota.

Küçük, F., \& Livanelioglu, P. T. (2015). Impact of the clinical Pilates exercises and verbal education on exercise beliefs and psychosocial factors in healthy women. Journal of Physical Therapy Science, 27(11), 34373443. doi: $10.1589 /$ jpts. 27.3437

Kuoppala, J., Lamminpaa, A., \& Husman, P. (2008). Work health promotion, job well-being, and sickness absences: A systematic review and meta-analysis. Journal of Occupational and Environmental Medicine, 50(11), 1216-1227. doi: 10.1097/JOM.0b013e31818dbf92

Leandro-Rojas, M. (2014). Potencial del espacio público como facilitador de bienestar y salud mental. Revista Costarricense de Psicología, 33(1), 31-45.

Martín, G. (2003). La mujer futbolista desde la perspectiva psicológica. Cuadernos de Psicología del Deporte, $3(2), 7-15$.

Martín, M., Pastor, M. A., Castejón, J., Lledó, A., López, S., Terol, M. C., \& Rodríguez-Marín, J. (2002). Valoración preliminar de la escala de Autoeficacia General en una muestra española. Revista de Psicología Social Aplicada, 12(2), 53-65.

McAuley, E., Courneya, K., Rudolph, D., \& Lox, C. (1994). Enhancing exercise adherence in middle-aged males and females. Preventive Medicine, 23(4), 498-506.

McCarthy, J. D., \& Hoge, D. R. (1982). Analysis of age effects on longitudinal studies of adolescent self-esteem. Developmental Psychology, 18, 372-379. doi: 10.1037/0012-1649.18.3.372

Meliá, J. L., \& Peiró, J. M. (1989). El Cuestionario de Satisfacción S10/12: Estructura factorial, fiabilidad y validez. Revista de Psicología del Trabajo y de las Organizaciones, 4(11), 179-187.

Memmedova, K. (2015). Impact of Pilates on axiety attention, motivation, cognitive function and achievement of students: Structural modelling. Social and Behavioral Sciences, 186, 544-548. doi: 10.1016/j. sbspro.2015.04.009

Mikkelsen, K., Stojanovska, L., Polenakovic, M., Bosevski, M., \& Apostolopoulos, V. (2017). Exercise and mental health. Maturitas, 106, 48-56. doi: https://doi.org/10.1016/j.maturitas.2017.09.003

Näswall, K., Hellgren, J., \& Sverke, M. (2008). The individual in the changing working life. Cambridge: Cambridge University Press.

Ooijendijk, W. T. M., Hildebrandt, V. H., \& Hopman-Rock, M. (2006). Physical activity measured 2002-2004. Eindhoven: TNO Quality of Lie.

Organización Mundial de la Salud. (2010). Recomendaciones mundiales sobre actividad física para la salud. Ginebra: Organización Mundial de la Salud. 
Otero, J. M., Luengo, A., Romero, E., Gómez, J. A., \& Castro, C. (1998). Psicología de la personalidad. Manual de prácticas. Barcelona: Ariel Practicum.

Pancorbo, A. E., \& Pancorbo, E. L. (2011). Actividad fisica en la prevención y tratamiento de la enfermedad cardiometabólica. La dosis del ejercicio saludable. Madrid: IMC.

Pérez-Marín, M. L., Yélamos, F., \& Rodríguez-Pérez, M. A. (2015). Intervención con un programa de Ejercicio Físico en la empresa. Medicina y Seguridad del Trabajo, 61(240), 342-353.

Pérez-Samaniego, V., \& Devís, J. (2003). La promoción de la actividad física relacionada con la salud. La perspectiva de proceso y de resultado. Revista Internacional de Medicina y Ciencias de la Actividad Física $y$ del Deporte, 3(10), 69-74.

Pilates, J. (1934). Your Health. Incline Village, Nevada: Presentation Dynamics Incorporated.

Pilates, J., \& Miller, W. (1945). Pilates return to life trough Contrology. Incline Village, Nevada: Presentation Dynamics Incorporated.

Raffo, E., Ráez, L., \& Cachay, O. (2013). Riesgos psicosociales. Revista de la Facultad de Ingeniería Industrial, 16(2), 70-79.

Reche, C., Cepero, M., \& Rojas, F. J. (2010). Efecto de la experiencia deportiva en las habilidades psicológicas de esgrimistas de ranking nacional español. Cuadernos de Psicología del Deporte, 10(2), 33-42.

Rosenberg, M. (1965). Society and the adolescent self-image. New Jersey: Princeton University Press.

Rueda, B., \& Pérez-García, A. M. (2004). Análisis comparativo de la competencia percibida general y la específica de salud. Ansiedad y Estrés, 10, 127-139.

Salanova, M., Schaufeli, W. B., Llorens, S., Peiró, J. M., \& Grau, R. (2000). Desde el "burnout" al "engagement”: ¿una nueva perspectiva? Revista de Psicología del Trabajo y las Organizaciones, 16(2), 117-134.

Sánchez, P. A., Ureña, F., \& Garcés de Los Fayos, E. J. (2002). Repercusiones de un programa de actividad física gerontológica sobre la aptitud física, autoestima, depresión y afectividad. Cuadernos de Psicología del Deporte, 2(2), 57-73.

Schaufeli, W., \& Salanova, M. (2002). ¿Cómo evaluar los riesgos psicosociales en el trabajo? Revista de Prevención, Trabajo y Salud, 20, 4-9.

Schaufeli, W. B., Salanova, M., González-Romá, V., \& Bakker, A. (2002). The measurement of engagement and burnout: A two sample confirmatory factor analytic approach. Journal of Happiness Studies, 3(1), 71-92.

Scheier, M. F., Carver, C. S., \& Bridges, M. W. (1994). Distinguishing optimism from neuroticism (and trait anxiety, self-mastery, and self-esteem): A reevaluation of the Life Orientation Test. Journal of Personality and Social Psychology, 67(6), 1063-1078.

Shea, S., \& Moriello, G. (2014). Feasibility and outcomes of a classical Pilates program on lower extremity strength, posture, balance, gait, and quality of life in someone with impairments due to a stroke. Journal of Bodywork and Movement Therapies, 18(3), 332-360. doi: 10.1016/j.jbmt.2013.11.017 
Solanes, A., Martín del Río, B., Rodríguez, K., Benavides, G., \& Martínez, F. (2013). Factores de riesgo psicosocial laboral y burnout en función de la categoría profesional. Revista de Psicología de la Salud (New Age), 1(1), 74-93.

Special Eurobarometer 412. (2014). Sport and Physical Activity. Recuperado de http://ec.europa.eu/health/ nutrition physical activity/docs/ebs 412 en.pdf

Stan, D. L., Collins, N. M., Olsen, M. M., Croghan, I., \& Pruthi, S. (2012). The evolution of mindfulness-based physical interventions in breast cancer survivors. Evidence Based Complementary and Alternative Medicine, Article ID 758641. doi: 10.1155/2012/758641

Stein, A. C., Molinero, O., Salguero, A., Corrêa, M. C. R., \& Márquez, S. (2013). Actividad física y salud en pacientes con enfermedad coronaria. Cuadernos de Psicología del Deporte, 14(1), 109-116.

U. S. Departament of Health and Human Services (2008). Physical activity guidelines for Americans. Washington: U. S. Departament of Health and Human Services.

Vázquez, A. J., Jiménez, R., \& Vázquez-Morejón, R. (2004). Escala de autoestima de Rosenberg: fiabilidad y validez en población clínica española. Apuntes de Psicología, 22(2), 245-257.

Recibido: 23 de Noviembre de 2016

Revisión recibida: 31 de Julio de 2018

Aceptado: 05 de Septiembre de 2018

\section{Sobre la autora y los autores:}

Salvador Boix Vilella es Doctor por la Universidad Miguel Hernández de Elche (España). Labora como profesor en la Facultad de Humanidades y Ciencias Sociales de la Universidad Isabel I (España).

Eva León Zarceño es Doctora en Psicología por la Universitat de València (España). Trabaja como docente del Departamento de Psicología de la Salud en la Universidad Miguel Hernández de Elche (España).

Miguel Ángel Serrano Rosa es Doctor en Psicología por la Universitat de València (España). Se desempeña como docente del Departamento de Psicobiología de la Universitat de València.

Publicado en línea: 20 de diciembre de 2018 